\title{
Kinetic tracking of Plasmodium falciparum antigen presentation reveals determinants of protein export and membrane insertion
}

Short title: Malaria antigens RISE to the erythrocyte surface

Jinfeng Shao ${ }^{1}$, Gunjan Arora ${ }^{2}$, Javier Manzella-Lapeira², Joseph A. Brzostowski², and Sanjay A. Desai $^{1, *}$

${ }^{1}$ Laboratory of Malaria and Vector Research and ${ }^{2}$ Laboratory of Immunogenetics, National Institute of Allergy and Infectious Diseases, National Institutes of Health, Rockville, MD, USA

*Address correspondence to Sanjay A. Desai, sdesai@,niaid.nih.gov 


\section{Abstract}

2 Intracellular malaria parasites export many proteins into their host cell, inserting several into the

3 erythrocyte plasma membrane to enable interactions with their external environment. While

4 static techniques have identified some surface-exposed proteins, other candidates have eluded

5 definitive localization and membrane topology determination. Moreover, both export kinetics

6 and the mechanisms of membrane insertion remain largely unexplored. We introduce Reporter

7 of Insertion and Surface Exposure (RISE), a method for continuous nondestructive tracking of

8 antigen exposure on infected cells. RISE utilizes a small 11 aa NanoLuc fragment inserted into a

9 target protein and detects surface exposure through high-affinity complementation. We tracked

10 insertion of CLAG3, a malaria parasite protein linked to nutrient uptake, throughout the $P$.

11 falciparum cycle in human erythrocytes. Our approach also revealed key determinants of

12 trafficking and surface exposure. Removal of a C-terminal transmembrane domain aborted

13 export. Unexpectedly, certain increases in the exposed reporter size improved surface exposure

14 by up to 50 -fold, revealing that both size and charge of the extracellular epitope influence

15 membrane insertion. Insertion of parasite proteins at the host cell surface and antigen

16 accessibility is regulated by multiple factors, enabling intracellular parasite survival and immune

17 evasion under a broad range of conditions. 


\section{Introduction}

Many viral, bacterial and parasitic microbes invade, grow, and replicate within host cells to evade immune detection, access host cell machinery for replication, and use cellular macromolecules as nutrient sources [1]. At the same time, the intracellular milieu limits the pathogen from accessing plasma nutrients and often provides an inhospitable ionic composition or acidic $\mathrm{pH}$. To overcome these hurdles, intracellular pathogens often export effector proteins into their host cell to remodel their abode, altering host cell defenses and physiology to their benefit [2-4]. A subset of effector proteins then insert in the host membrane to enable pathogen interactions with the extracellular space. These exposed proteins serve diverse roles and are acknowledged vaccine and drug targets.

In the virulent human malaria parasite Plasmodium falciparum, surface-exposed proteins benefit pathogen replication by facilitating cytoadherence [5], immune evasion [6] and nutrient uptake [7]. These antigens have been identified through static assays such as confocal and electron microscopy techniques. Because these methods lack the required spatial resolution [8], they cannot unambiguously determine if some parasite proteins are surface-exposed or only adherent to the inner membrane face or cytoskeleton. In some cases, susceptibility to extracellular proteases or antibody-based assays with live cells can resolve this uncertainty $[9,10]$, but these approaches also have limitations. Another approach, mass spectrometry-based identification after surface labeling with NHS esters [11], has yielded a largely unvalidated list of proteins that is complicated by increased NHS ester permeability after infection with

Plasmodium spp. [12].

Currently available methods also suffer from an inability to track the timing and kinetics of antigen insertion at the host membrane, hindering molecular insights. Both surface exposure via 
41 fusion of exocytic vesicles and "punch-through" insertion of soluble protein into the host

42 membrane have been proposed $[13,14]$, but the absence of direct and quantitative measurements

43 has prevented definitive mechanistic insights.

44 To address these limitations and better define how pathogen proteins insert at their host cell membrane, we developed and used a Reporter of Insertion and Surface Exposure (RISE). Our study combines RISE with biochemical studies of the target reporter protein to identify constraints on protein trafficking and membrane insertion.

\section{Results}

HiBiT tagging within a conserved surface antigen in malaria parasites

We sought to generate a sensitive kinetic reporter for protein insertion on infected cells and

52 chose human erythrocytes infected with the virulent $P$. falciparum malaria parasite. Indirect

53 immunofluorescence microscopy assays (IFA) with antibodies against exposed epitopes offer a

54 specific readout but suffer from low spatial resolution and provide limited kinetic information

55 about protein export and host membrane insertion. Split enzyme reporters can overcome these

56 limitations when one enzyme fragment is introduced into the exported protein; a specific signal

57 is produced through complementation with a second fragment added extracellularly. Similar

58 location-specific complementation has been described using mammalian proteins [15], but this

59 approach has not been used to track appearance of pathogen-derived antigens on host cells.

60 Because it relies on extracellular interactions with a surface-exposed epitope, our strategy

61 resembles antigen presentation on immune effector cells [16].

62 The bright NanoLuc luciferase is an ideal enzymatic reporter for bloodstage P. falciparum

63 studies [17] and has recently been optimized for development of a split reporter [18]. We 
selected the 11 residue HiBiT and $18 \mathrm{kDa} \mathrm{LgBiT}$ fragments of NanoLuc for our studies, based on their strong association $\left(K_{D}=700 \mathrm{pM}\right)$ that yields an ATP-independent, furimazine-sensitive luminescence signal.

We next reasoned that minimal modification of a normally exported parasite protein would be more informative about parasite biology than extensively engineered reporters, as used previously [19]. We preferred the parasite CLAG3 protein for these studies over antigens such as PfEMP1 and RIFINs encoded by large multigene families to avoid epigenetic regulation and variable expression [20]. Clag paralogs also undergo epigenetic silencing [21], but some clones carry a single constitutively expressed hybrid clag3 gene termed clag3h [22]. One such line, $\mathrm{KC5}$, has been successfully used for transfections without the risk of epigenetic silencing [23]. CLAG3 is also the only known surface-exposed protein conserved in all examined Plasmodium spp. [24], suggesting that trafficking insights made using this protein may be broadly applicable. Because CLAG3 expression is linked to the plasmodial surface anion channel (PSAC), an ion and nutrient uptake channel at the host membrane [9], transport studies with transfectant parasites would also provide a biochemical correlate of reporter signal activity. We therefore selected CLAG3 for tagging and the KC5 clone for production of a surface exposure reporter parasite. CLAG3 has a small 10-30 aa hypervariable region that appears to be exposed at the host membrane (HVR, Fig 1A) [25]. We therefore used CRISPR/Cas9 editing to replace the KC5 clag3h HVR sequence with a single HiBiT tag flanked by 8 aa linker sequences; we named this limiting dilution clone $8-1$ based on the size of the flanking linker and the number of inserted HiBiT tags (Fig 1A, bottom). HVR replacement increased the size of the extracellular loop domain by a modest 10 residues (S1A Fig). We also produced $8-1 H A$, a similar line with an HA epitope tag added after the HiBiT linker cassette. Two additional lines, 8-1trunc and 8- 
87 1HAtrunc, express truncated CLAG3 reporters with a stop codon introduced after the inserted cassette (Fig 1A, bottom and S1A Fig). We initially reasoned that these truncation constructs would yield a more flexible extracellular HiBiT epitope and provide insights into roles served by the downstream CLAG3 sequence. Although CLAG3 is a critical determinant of PSAC activity, CLAG3 knockout parasites are viable [26], possibly because other CLAG paralogs in $P$. falciparum compensate for CLAG3 loss. Thus, we expected that our modifications would be tolerated unless they produce a dominant-negative effect on nutrient uptake [27].

Immunoblotting with each cloned transfectant confirmed expression and revealed single bands of expected size. Probing with anti-CLAG3 confirmed loss of this antibody's C-terminal epitope in the 8-1trunc clone and unchanged electrophoretic migration in $8-1$ (Fig 1B, top). Both engineered CLAG3 isoforms were identified using a LgBiT probe that binds to HiBiT-tagged proteins to produce a luminescence signal (Fig 1B, bottom); the KC5 parent was not recognized by $\mathrm{LgBiT}$, confirming specificity of this probe for the HiBiT tag. Similar results were obtained in the HA tandem-tagged parasites (Fig 1C), establishing faithful expression.

IFA confirmed and extended these findings. At the schizont stage, we detected each variant shortly after stage-specific synthesis under the genomic clag3h promoter (Fig 1D, upper group of images). While 8-1 HA parasites trafficked the modified CLAG3 protein normally to developing rhoptries, the truncated tagged protein in 8 -1HAtrunc produced a more diffuse pattern with a small fraction reaching the rhoptry to colocalize with RhopH3, an associated protein that also contributes to PSAC formation [28].

At merozoite egress and reinvasion, rhoptry proteins are secreted into the next erythrocyte and deposited into the parasitophorous vacuole [29]. From there, through an incompletely understood interaction with the PTEX translocon, CLAG3 is exported into host cytosol for 
trafficking to the host membrane $[28,30]$. Imaging revealed that the tandem-tagged CLAG3 protein in $8-1 H A$ trafficked as expected and colocalized with RhopH3 at the host cell surface

112 (Fig 1D, lower group); an antibody specific for the CLAG3 c-terminus further confirmed this

113 localization (S1B Fig). In contrast, the truncated CLAG3 in 8-1HAtrunc parasites was less

114 abundant, suggesting that its poor trafficking to rhoptries compromised delivery to the next

115 erythrocyte upon reinvasion. The small pool of this protein delivered into trophozoites failed to be exported and did not colocalize with RhopH3 (Fig 1D, bottom row). trophozoite parasites contained lower amounts that reflect incomplete transfer from prior cycle schizonts during egress and reinvasion [28]. The truncated protein in 8-1HAtrunc was detected

122 in schizonts but not in ring- and trophozoite-stage parasites, further implicating a role of the 123 CLAG3 c-terminal region in efficient transfer to rhoptries and new erythrocytes during invasion. interactions for these CLAG3 reporter proteins (Fig 1F, silver-stained gel). CLAG3 was

127 lines (bands labeled "1"), confirming specific pull-down. RhopH2 and RhopH3, unrelated 128 proteins that interact with CLAG3 [24], were recovered from $8-1 H A$ (“2” and "3"), albeit with 129 lower efficiency than in experiments using CLAG3-tv2, an engineered control parasite that a 
133 yielded an unchanged RhopH2 band and a smaller band as expected for truncated CLAG3, but

134 RhopH3 was not detected in these silver-stained gels (Fig 1F). Immunoblotting confirmed

135 recovery of RhopH3 in 8-1HA pull-downs and failed interaction with RhopH3 upon CLAG3

136 truncation (Fig 1G). The recent cryo-EM RhopH complex structure reveals that CLAG3

137 interacts with RhopH3 via two primary domains termed the CLAG3 “300 region" and "1300

138 loop" [30]. Because the 1300 loop is distal to the site of CLAG3 truncation in 8-1HAtrunc,

139 these findings suggest that this loop is required for stable CLAG3-RhopH3 interaction.

Kinetics of membrane insertion and surface exposure

142

143

144

145

146

147

148

149

150

151

152

153

154

155

156

We next monitored stage-specific CLAG3 surface exposure on infected erythrocytes with the RISE method. We measured luminescence resulting from complementation of the HiBiT tag by extracellular LgBiT (Fig 2A). Bioluminescence microscopy revealed an undetectable reporter signal on immature ring-infected erythrocytes (Fig 2B, left panels) on KC5 and both HiBiT tagged lines, consistent with the appearance of PSAC activity on infected cells only after parasite maturation [32]. In contrast, trophozoite-infected cells exhibited a surface-distributed luciferase signal specific to $8-1$ (Fig 2B-C). The 8-1trunc parasite matured normally but failed to produce a surface signal.

We then miniaturized this reporter assay into 96-well microplate wells and tracked CLAG3 exposure kinetics in cultures initiated shortly after invasion. Although both clones exhibited negligible signals for the first $16 \mathrm{~h}$ of the parasite cycle, this lag was followed by a rapid increase in CLAG3 membrane insertion to produce luminescence on $8-1$ (Fig 2D, red circles). This signal reached a plateau between 30 and $44 \mathrm{~h}$ on $8-1$, during which $8-1$ trunc parasites continued to produce minimal luminescence. At the end of the erythrocyte cycle (44-48 h), both transfectant cultures exhibited abruptly increased signals, consistent with merozoite egress and CLAG3 
157 discharge into extracellular medium [31]. At the signal plateau, approximately $1 / 3$ of the

158 CLAG3 within 8-1 infected cells had become surface-exposed based on measured reporter signal

159 before and after detergent release (Fig 2E, $36 \mathrm{~h}$ timepoint).

160 Prior studies of the CLAG3 HVR implicated exposure at the host cell surface based on this

161 motif's susceptibility to extracellular protease $[9,25]$. We therefore examined whether the

162 exposed HiBiT is also susceptible to external protease by measuring luminescence signals after a

163 brief protease treatment of trophozoite infected cells. While the background signal in KC5

164 parasites and the low-level signal from 8-1trunc cells were not significantly affected by

165 extracellular protease treatment $(P>0.1, n=5$ independent trials each; Fig $2 \mathrm{~F})$, the large signal

166 produced by $8-1$ infected cells was reduced by $39 \pm 3 \%$ upon treatment with extracellular

167 protease $(P=0.005, n=5)$, further confirming that our split NanoLuc assay faithfully reports on

168 CLAG3 exposure at the host cell surface.

\section{Failed export compromises channel-mediated permeability}

171 We next examined the effects of these CLAG3 modifications on nutrient uptake at the host

172 membrane. We tracked uptake of sorbitol, a sugar alcohol with high PSAC permeability, and

173 found that both the $8-1$ and 8 -1trunc parasites increase host cell permeability (Fig 3A), as

174 expected from its requirement for intracellular pathogen survival $[27,28]$. Both $8-1$ and $8-1$ trunc

175 parasites exhibited lower sorbitol permeabilities than the parental KC5 (Fig 3B, $P<10^{-4}, n=20$ -

17621 trials each, one-way ANOVA with post-hoc tests), but uptake was preserved to a greater

177 extent in $8-1$. The reduced permeability in 8-1trunc matched that of a recently reported CLAG3

178 knockout, $C 3 h-K O(P=0.55$; ref \# [26]), indicating that the truncated CLAG3 in this parasite 
179 does not measurably contribute to PSAC activity. Failure to traffic and insert this protein in the

180

181

182

183

184

185

186

187

188

189

190

191

192

193

194

195

196

197

198

199

200

201

host membrane conservatively accounts for this phenotype (Fig 2).

CLAG3 cleavage within the HVR by extracellular protease compromises solute transport [25]. Here, we found that pronase E treatment reduced channel-mediated transport in $\mathrm{KC} 5$ and 8-1, but had no effect in 8-1trunc parasites (red traces, Fig 3A; Fig 3C, $P<10^{-4}, n=10-11$ trials, one way ANOVA with post-hoc tests), also consistent with intracellular retention of the truncated CLAG3 protein.

Immunoblots using an antibody directed against the CLAG3 C-terminus revealed single $\sim 37$ $\mathrm{kDa}$ cleavage products in KC5 and $8-1$ (Fig 3D), corresponding to proteolysis at the surfaceexposed HVR and release of the distal fragment. Cleaved band intensities revealed that these two parasites exported and inserted CLAG3 protein at the host membrane with indistinguishable efficacies (Fig 3D, bar graph). Because 8-1trunc parasites express a truncated CLAG3 not recognized by this antibody, we then probed these blots with LgBiT. This approach is based on visualization of HiBiT-tagged proteins via the luminescence generated upon LgBiT complementation. This treatment reduced the band intensity in protein from $8-1$ parasites but had negligible effect on 8-1trunc CLAG3 (Fig 3E, bar graph), consistent with proteolytic degradation of an exposed HiBit tag only on 8-1 parasites.

These studies establish that CLAG3 must insert at the host membrane to contribute to PSAC activity because the truncated protein in 8 -1trunc parasites has transport activity matching that of a CLAG3-null parasite. They also reveal that proteolysis at a surface-exposed loop on CLAG3 compromises transport regardless of sequence as this site retained its susceptibility when replaced by a HiBiT reporter. 


\section{Membrane insertion not compromised by increased extracellular loop size}

203

204

205

206

207

208

209

210

To examine possible constraints on insertion of the CLAG3 HVR at the host membrane, we generated an additional transfectant carrying a larger 3xHA epitope tag after the HiBiT cassette (S1C Fig). Biochemical studies with $8-1 H A$ and this new parasite, $8-1-3 H A$, revealed marked increases in luminescence signals as the extracellular loop size increased by 9 and 27 residues, respectively. Using matched numbers of trophozoite-stage parasites, we found a 7- and 50-fold higher luminescence signals from the $8-1 H A$ and $8-1-3 H A$ parasites (Fig 4A, red bars) than from 8-1. The increases in luminescence were more modest when measured after cell lysis with detergent (black bars). Greater accentuation with intact cells than after lysis is consistent with steric hindrance or constrained HiBiT presentation in a minimal extracellular loop, as proposed for CLAG3 [25]. Addition of these tags also significantly increased susceptibility of the HiBiT reporter to extracellular protease, with the larger $3 \mathrm{xHA}$ tag producing a greater reduction in luminescence upon protease treatment (Fig 4B). Membrane insertion and surface exposure were further confirmed with immunoblotting (Fig 4C) and luminescence imaging, which revealed dramatically increased signals from intact cells (Fig 4D). These findings suggest improved LgBiT binding and reporter complementation upon adjacent HA epitope tagging, presumably because the size and negative charge of this tag improves accessibility at the extracellular loop and within soluble RhopH complexes upon detergent release.

These larger insertions into CLAG3 had modest effects on channel-mediated sorbitol uptake at the host membrane (Fig 4E). The resulting channels also retained quantitatively similar protease susceptibilities (Fig 4F), consistent with minimally affected CLAG3 insertion and PSAC formation at the host membrane.

\section{Larger epitopes reveal complex regulation of CLAG3 membrane insertion}


To further explore size and charge constraints on pathogen epitope presentation, we made

227

228

229

230

231

232

233

234

235

236

237

238

239

240

241

242

243

244

245

246

247

248

additional transfectants containing multiple HiBiT tags with two different linker sizes (Fig 5A).

As these constructs retained upstream and distal CLAG3 sequences, each modified protein

trafficked normally through schizonts and was delivered into maturing trophozoite-infected cells

(S2A-B Fig), where the increased size of the targeted protein was apparent in immunoblots (Fig

5B). Although immunofluorescence, sorbitol permeability measurements and protease

susceptibility studies all suggested that CLAG3 failed to export and undergo host membrane

insertion to enable PSAC activity in these lines (S2B Fig and Fig 5C), bioluminescence intensity

analyses using RISE identified individual cells that presented CLAG3 on host cells (Fig 5D).

Notably, while most cells in each of the largest multiple HiBiT constructs produced background

signals, a few cells produced very bright signals that exceeded those seen on $8-1$ parasites.

These intense signals may reflect either reduced steric hindrance with larger, more flexible

extracellular loops or signal amplification from HiBiT multiplicity on each protein. The

markedly differing signals from individual cells is unexpected for these clonal lines. This

observation suggests epigenetic control of CLAG3 export and host membrane insertion.

We tabulated the HVR sequences from 38 available CLAG3 sequences and compared their

properties to those of the lines we have engineered. Although they are variant, the native HVR sequences tended to have a modest net negative charge (Fig 5E, black symbols). In contrast, the constructs containing more than one HiBit epitope were increasingly basic, yielding net positive charges on the at the extracellular loop (red symbols). Notably, the sequence in $8-1-3 H A$, whose CLAG3 successfully inserted at the host membrane to produce remarkably bright luminescence using our RISE assays, had a higher molecular weight than that of $8-2$, which failed to traffic CLAG3 protein faithfully in most cells. It appears that the domain's net positive charge in $8-2$ 
and other multiple HiBit lines prevents trafficking and host membrane insertion. Along with structural constraints that ensure CLAG3-mediated nutrient uptake and evolutionary pressures to evade host immunity, the extracellular loop of this conserved protein family must also meet charge and size requirements for faithful trafficking and host membrane insertion.

\section{Discussion}

We present a new reporter that detects insertion of pathogen virulence antigens on their host cells. Our use of a split NanoLuc reporter is broadly applicable to a range of intracellular pathogens and will permit non-destructive kinetic tracking of antigens at the host cell surface. Some proteins targeted to underlying membranes, such as the parasitophorous vacuolar membrane of Plasmodia, Toxoplasma, and other parasites, may also be studied using selective permeabilization of the host membrane [33].

This reporter assay represents an important step toward understanding how pathogens interact with their host cells and will provide quantitative insights into the presentation of targeted antigens to the host immune system. We used this new technology to examine presentation of the conserved CLAG3 antigen on the surface of human erythrocytes. Associated nutrient channel transport, protein chemistry, and bioluminescence confocal microscopy studies all validated our new method.

Our findings implicate a revised model of CLAG3 trafficking. CLAG3 produced in schizonts remains inaccessible to extracellular LgBiT upon transfer to ring-infected cells; as the intracellular parasite matures, the protein is exported and inserts in the host membrane with kinetics that parallel the gradual appearance of the associated nutrient channel activity on trophozoite-infected cells. A C-terminal truncation that does not alter the reporter or its 
272 upstream sequence compromised trafficking, abolished host membrane insertion, and reduced

273 host cell permeability to levels seen in a recently reported CLAG3-null parasite. Using varied

274 reporter insertions, we also uncovered complexities in this protein's insertion into the erythrocyte

275 membrane; our studies suggest that both size and charge of the extracellular peptide loop

276 determine host membrane insertion.

277 Our luminescence imaging studies revealed marked variation between cells in cloned

278 multiple HiBit reporter lines, with some cells exhibiting high levels of CLAG3 exposure despite

279 insertion of large reporter domains. This finding implicates an epigenetic, post-translational

280 mechanism for regulating surface exposure. We propose that this may reflect altered expression

281 of one or more parasite chaperone proteins in host cytosol [34] or post-translational

282 modifications of CLAG3 [35,36]. Epigenetic control of antigen presentation on infected cells

283 has, to our knowledge, not been proposed for any intracellular pathogens. This finding reveals

284 the remarkable sophistication of malaria parasites in controlling their interactions with host

285 plasma and further promotes immune evasion.

286 We envision that the quantitative and sensitive readout enabled by a small HiBiT epitope

287 inserted at exposed antigen sites will unveil how pathogens modify their host cells while evading

288 immune attack.

\section{Materials and Methods}

291 Parasite cultivation and transfection

292 The $P$. falciparum $\mathrm{KC} 5$ parasite clone and its engineered derivatives were cultivated in $\mathrm{O}+$

293 human erythrocytes (Interstate Blood Bank, Inc.) at 5\% hematocrit in standard RPMI 1640-based

294 media (KD medical) supplemented with supplemented with $25 \mathrm{mM}$ HEPES, $50 \mu \mathrm{g} / \mathrm{mL}$ 

hypoxanthine, $0.5 \%$ NZ Microbiological BSA (MP Biomedicals), gentamicin and $28.6 \mathrm{mM}$ $\mathrm{NaHCO}_{3}\left(\right.$ Gibco) at $37^{\circ} \mathrm{C}$ under $90 \% \mathrm{~N}_{2}, 5 \% \mathrm{CO}_{2}, 5 \% \mathrm{O}_{2}$.

CRISPR-Cas9 DNA transfection of parasites to produce reporter lines was performed using electroporation of pUF1-Cas9 and modified pL6 plasmids into uninfected erythrocytes as described previously [28]. Plasmids were constructed using synthetic double-stranded DNA (Integrated DNA Technologies) and In-Fusion cloning (Takara) into the pL6 plasmid. Single guide RNAs (sgRNA), selected using on-, off- and paralog specificity scores [37], were also introduced using In-Fusion. After erythrocyte electroporation and addition of schizont-staged parasites, the culture was selected with 1.5 $\mu \mathrm{M}$ DSM1 and 2.5 nM WR99210. After parasite outgrowth and PCR confirmation of integration, limiting dilution cloning was performed for all transfectant lines. All experiments were performed with sequence-verified clones.

\section{Immunoblotting}

Synchronous parasite cultures were harvested, percoll-enriched where indicated, and used for immunoblotting experiments after hypotonic lysis $\left(7.5 \mathrm{mM} \mathrm{Na}_{2} \mathrm{HPO}_{4}, 1 \mathrm{mM}\right.$ EDTA, $1 \mathrm{mM}$ PMSF, pH 7.5) and solubilization in Laemmli sample buffer containing 6\% SDS. When required, samples were matched with turbidity measurements at $700 \mathrm{~nm}$. Proteins were separated by SDS-PAGE (4-15\% Mini-PROTEAN TGX gel, Bio-RAD) and transferred to nitrocellulose membranes. After blocking with 3\% skim milk powder in $150 \mathrm{mM} \mathrm{NaCl}, 20 \mathrm{mM}$ TrisHCl, pH 7.4 with $0.1 \%$ Tween 20 at $\mathrm{RT}$ for $1 \mathrm{~h}$, primary antibodies were applied in the same blocking buffer at a 1:1000-1:3000 dilution and incubated overnight at $4{ }^{\circ} \mathrm{C}$ with gentle rocking. After three washes in $150 \mathrm{mM} \mathrm{NaCl}, 20 \mathrm{mM}$ TrisHCl, pH 7.4 with $0.1 \%$ Tween20, HRPconjugated secondary antibodies were added at 1:3000 dilution. The blot was incubated for $1 \mathrm{~h}$ 
and washed three times. Imaging was performed after addition of Clarity Western ECL substrate (Bio-Rad) using the AI 680 imager (GE healthcare) or standard x-ray film exposure.

Where used, protease treatment was performed after washing and resuspending enriched trophozoite-infected cells in PBS-2 $\left(150 \mathrm{mM} \mathrm{NaCl}, 20 \mathrm{mM} \mathrm{Na}_{2} \mathrm{HPO}_{4}, 0.6 \mathrm{mM} \mathrm{CaCl}_{2}, 1 \mathrm{mM}\right.$ $\mathrm{MgCl}_{2}, \mathrm{pH} 7.4$ ) at $5 \%$ hematocrit with $1 \mathrm{mg} / \mathrm{mL}$ pronase $\mathrm{E}$ (Sigma) for $45-60 \mathrm{~min}$ at $37{ }^{\circ} \mathrm{C}$. The treated cells and matched untreated cells were washed in PBS-2 with $1 \mathrm{mM}$ PMSF at $4{ }^{\circ} \mathrm{C}$ before an additional wash in this buffer with $1 \mathrm{mM}$ EDTA. After hypotonic lysis, the membrane fraction was harvested by ultracentrifugation $\left(100,000 \times g, 1 \mathrm{~h}\right.$ at $\left.4{ }^{\circ} \mathrm{C}\right)$ and solubilized in Laemmli sample buffer as above.

Blots probed with LgBiT used the HiBiT blotting system kit (Promega). After protein transfer, nitrocellulose membranes were washed in $150 \mathrm{mM} \mathrm{NaCl}, 20 \mathrm{mM}$ TrisHCl, pH 7.4 with 0.1\% Tween20. LgBiT was applied in this blotting buffer at 1:200 dilution and incubated overnight at $4{ }^{\circ} \mathrm{C}$ with gentle rocking. Furimazine was then added in blotting buffer at a 1:500 dilution before imaging as above.

Band intensities were quantified using Image $\mathrm{J}$ software. Statistical analyses were based on three independent trials.

\section{Coimmunoprecipitation}

Schizont-stage infected cells were percoll-sorbitol enriched and lysed with 20 volumes of $10 \mathrm{mM}$ Tris $\mathrm{pH} 7.5,300 \mathrm{mM} \mathrm{NaCl}, 1 \%$ Triton-X100, $1 \mathrm{mM}$ PMSF. After a $30 \mathrm{~min}$ incubation at $4{ }^{\circ} \mathrm{C}$, solubilized proteins were separated by centrifugation $\left(14,000 \mathrm{x} g, 15 \mathrm{~min}, 4^{\circ} \mathrm{C}\right)$ and incubated with anti-HA affinity agarose beads (Sigma) with gentle mixing overnight at $4{ }^{\circ} \mathrm{C}$. After five washes, bound protein was eluted by addition of $2.5 \mathrm{mg} / \mathrm{mL}$ HA peptide in $10 \mathrm{mM}$ Tris $\mathrm{pH} 7.5$, 
341

342

343

344

345

346

347

348

349

350

351

352

353

354

355

356

357

358

359

360

361

362

$250 \mathrm{mM} \mathrm{NaCl}, 0.1 \%$ Triton-X100 for $30 \mathrm{~min}$. Eluted proteins were resuspended in Laemmli sample buffer and subjected to SDS-PAGE.

\section{Immunofluorescence assays}

Indirect immunofluorescence assays were performed using air-dried thin smears after fixation with 1:1 acetone:methanol at $-20{ }^{\circ} \mathrm{C}$ for 2 min. Slides were then dried, blocked with $3 \%$ milk in PBS for $1 \mathrm{~h}$ at RT, and incubated with primary antibodies in blocking buffer (mouse antiCLAG3, 1:100; rabbit anti-RhopH3, 1:500; mouse anti-HA, 1:100) for $1.5 \mathrm{~h}$ at RT under coverslips. After two washes with chilled PBS, Alexa Fluor 488 or 594-conjugated secondary antibody at a 1:500 dilution and $10 \mu \mathrm{g} / \mathrm{mL}$ DAPI were added in blocking buffer and incubated for 30 min at RT. After washes and drying, slides were mounted with Prolong Diamond antifade mountant (Molecular Probes). Images were collected on a Leica SP8 microscope using a 64x oil immersion objective with serial $405 \mathrm{~nm}, 488 \mathrm{~nm}$, or $594 \mathrm{~nm}$ excitation. Images were processed using Leica LAS X and Huygens software.

\section{Enrichment of ring-infected cells}

Ring-stage cultures were further synchronized by a 20 min incubation in $4 \%$ xylitol, a sugar alcohol with high PSAC permeability [38], to lyse mature infected cells. The culture was then resuspended and incubated in culture medium supplemented with $207 \mathrm{mM}$ xylitol for $1 \mathrm{~h}$ at 37
${ }^{\circ} \mathrm{C}$. This cell suspension was then layered on a discontinuous percoll-xylitol gradient a bottom layer of $72 \%$ Percoll and an upper layer of $40 \%$ Percoll; both solutions were prepared in RPMI 1640 medium with $208 \mathrm{mM}$ xylitol, $12.4 \mathrm{mM}$ HEPES, and $16.3 \mathrm{mg} / \mathrm{L}$ BSA. After centrifugation 
$363\left(10,000 \times g\right.$ for $30 \mathrm{~min}$ at $\left.21^{\circ} \mathrm{C}\right)$, ring-stage infected cells at $65-90 \%$ parasitemia were harvested 364 and washed by dropwise addition of culture medium.

\section{Luminescence measurements and export kinetics}

367 Luminescence measurements were performed using percoll-enriched infected erythrocytes in 368384 well microplates using the Nano-Glo HiBiT extracellular detection system (Promega). Cells

369 were resuspended at $0.2 \%$ hematocrit in culture medium diluted with two volumes of 200:1:50

370 buffer:LgBiT:Furimazine, according to the manufacture's protocol. After a 30 min RT

371 incubation, luminescence was measured using the Centro XS3 LB 960 reader (Berthold) or

372 Synergy Neo2 (BioTek) with a counting time of $0.5 \mathrm{~s} /$ well.

373 CLAG3 export kinetics were tracked using luminescence after seeding enriched ring-infected

374 cells in culture medium at $0.5 \%$ hematocrit into triplicate wells at $60 \mu \mathrm{L} / w e l l$. The plates were

375 sealed with Breathe-Easy sealing membrane (RPI) and incubated at $37{ }^{\circ} \mathrm{C}$ under $5 \% \mathrm{CO}_{2}$ in air.

376 At timed intervals, $40 \mu \mathrm{L}$ of medium from selected wells was replaced with Nano-Glo HiBiT

377 extracellular buffer with LgBiT and furimazine substrate (Promega). Readings were taken after

378 a $30 \mathrm{~min}$ room temperature incubation as described above.

\section{Bioluminescence microscopy}

381 Erythrocytes infected with reporter parasite clones were imaged using a LV200 inverted

382 bioluminescence microscope with a temperature-controlled stage (Olympus). Enriched ring- or

383 trophozoite-infected erythrocytes were resuspended in culture medium at $2.5 \%$ hematocrit before

384 diluting 50x into Nano-Glo HiBiT extracellular buffer (Promega) with LgBiT and furimazine at

$385100 x$ and 50x dilutions, respectively for a total volume of $100 \mu \mathrm{L}$ in a $35 \mathrm{~mm}$ poly-D-lysine 
coated coverslip dish (MatTek). Cells were allowed to settle at $37^{\circ} \mathrm{C}$ for $25 \mathrm{~min}$ in the LV200 microscope before selecting fields of view for imaging. Bioluminescence images were collected with a 45 min exposure under a 64x oil immersion objective. Images were visualized and adjusted to 14 bit in LCmicro_2.2 software (Olympus).

Single cell luminescence intensities were quantified using a locally-developed macro that uses the corresponding brightfield image to define the cell boundary. This macro reports luminescence intensities over the cell, tabulating mean, max and min values along with the cell $2 \mathrm{D}$ area and is available upon request.

\section{Osmotic lysis assays}

The kinetics of PSAC-mediated sorbitol uptake and infected cell osmotic lysis were continuously tracked as described previously [26]. Enriched trophozoite-stage infected cells were washed and resuspended in $150 \mathrm{mM} \mathrm{NaCl}, 20 \mathrm{mM}$ Na-HEPES buffer, $0.1 \mathrm{mg} / \mathrm{mL}$ BSA, $\mathrm{pH}$ 7.4. Solute uptake was initiated by the addition of $280 \mathrm{mM}$ sorbitol, $20 \mathrm{mM}$ Na-HEPES, $0.1 \mathrm{mg} / \mathrm{mL}$ BSA, $\mathrm{pH}$ 7.4. Concomitant uptake of sorbitol and water produces osmotic lysis at rates directly proportional to PSAC sorbitol permeability. Lysis kinetics were continuously monitored using transmittance of $700 \mathrm{~nm}$ light through the cell suspension. Osmotic lysis half-times, normalized permeability estimates, and measures of protease effect were determined from the recordings with locally developed code.

\section{Computational and statistical analyses}

CLAG3 sequences were downloaded from www.plasmodb.org and aligned using Multiple Sequence Alignment (MUSCLE) to identify HVR sequences from available $P$. falciparum 
409 paralogs. The molecular weight and net charge at $\mathrm{pH} 7.4$ was calculated at

410 http://protcalc.sourceforge.net/.

411 Numerical data are shown as mean \pm S.E.M. Data were analyzed in SigmaPlot 10.0 (Systat)

412 or Prizm 8 (GraphPad). Statistical significance was determined using unpaired or paired

413 Student's $t$-test or one-way ANOVA with post hoc Tukey's multiple comparisons test as

414 appropriate. Significance was accepted at $P<0.05 \%$.

415

416

417

418

\section{Acknowledgements}

420 We thank Gagan Saggu for help with immunofluorescence assays and David Jacobus for

421 WR99210. DSM1 (MRA-1161) was obtained through MR4 as part of the BEI Resources

422 Repository, NIAID, NIH.

423 


\section{Author contributions}

425 Conceptualization: Jinfeng Shao, Sanjay A. Desai.

426 Data curation: Jinfeng Shao, Gunjan Arora, Javier Manzella-Lapeira, Joseph A. Brzostowski,

427 Sanjay A. Desai.

428 Formal analysis: Jinfeng Shao, Gunjan Arora, Javier Manzella-Lapeira, Joseph A. Brzostowski,

429 Sanjay A. Desai.

430 Investigation: Jinfeng Shao, Gunjan Arora, Javier Manzella-Lapeira.

431 Supervision: Sanjay A. Desai.

432 Writing - original draft: Jinfeng Shao, Sanjay A. Desai.

433 Writing - review \& editing: Jinfeng Shao, Gunjan Arora, Javier Manzella-Lapeira, Joseph A.

434 Brzostowski, Sanjay A. Desai.

435

436 Competing interests

437 The authors declare no competing interests. 


\section{References}

1. Thakur A, Mikkelsen H, Jungersen G. Intracellular pathogens: host immunity and microbial persistence strategies. J Immunol Res. 2019; 2019:1356540. PMID: 31111075

2. Cornejo E, Schlaermann P, Mukherjee S. How to rewire the host cell: a home improvement guide for intracellular bacteria. J Cell Biol. 2017; 216: 3931-48. PMID: 29097627

3. Marti M, Baum J, Rug M, Tilley L, Cowman AF. Signal-mediated export of proteins from the malaria parasite to the host erythrocyte. J Cell Biol. 2005; 171: 587-92. PMID: 16301328

4. Laliberte J, Carruthers VB. Host cell manipulation by the human pathogen Toxoplasma gondii. Cell Mol Life Sci. 2008; 65: 1900-15. PMID: 18327664

5. Lee WC, Russell B, Renia L. Sticking for a cause: The falciparum malaria parasites cytoadherence paradigm. Front Immunol. 2019; 10:1444. PMID: 31316507

6. Yam XY, Preiser PR. Host immune evasion strategies of malaria blood stage parasite. Mol Biosyst. 2017; 13: 2498-508. PMID: 29091093

7. Desai SA. Why do malaria parasites increase host erythrocyte permeability? Trends Parasitol. 2014; 30: 151-9. PMID: 24507014

8. Mayhew TM, Lucocq JM. Developments in cell biology for quantitative immunoelectron microscopy based on thin sections: a review. Histochem Cell Biol. 2008; 130: 299-313. PMID: 18553098

9. Nguitragool W, Bokhari AA, Pillai AD, Rayavara K, Sharma P, Turpin B et al. Malaria parasite clag3 genes determine channel-mediated nutrient uptake by infected red blood cells. Cell 2011; 145: 665-77. PMID: 21620134

10. Jouin H, Goguet de la Salmoniere YO, Behr C, Huyin Qan DM, Michel JC, Sarthou JL et al. Flow cytometry detection of surface antigens on fresh, unfixed red blood cells infected by Plasmodium falciparum. J Immunol Methods. 1995; 179: 1-12. PMID: 7868917

11. Florens L, Liu X, Wang Y, Yang S, Schwartz O, Peglar M et al. Proteomics approach reveals novel proteins on the surface of malaria-infected erythrocytes. Mol Biochem Parasitol. 2004; 135: 1-11. PMID: 15287581

12. Cohn JV, Alkhalil A, Wagner MA, Rajapandi T, Desai SA. Extracellular lysines on the plasmodial surface anion channel involved in $\mathrm{Na}^{+}$exclusion. Mol Biochem Parasitol. 2003; 132: 27-34. PMID: 14563534

13. Taraschi TF, Trelka D, Martinez S, Schneider T, O'Donnell ME. Vesicle-mediated trafficking of parasite proteins to the host cell cytosol and erythrocyte surface membrane in Plasmodium falciparum infected erythrocytes. Int J Parasitol. 2001; 31: 1381-91. PMID: 11566305 
14. Papakrivos J, Newbold CI, Lingelbach K. A potential novel mechanism for the insertion of a membrane protein revealed by a biochemical analysis of the Plasmodium falciparum cytoadherence molecule PfEMP-1. Mol Microbiol. 2005; 55: 1272-84. PMID: 15686570

15. Wehrman TS, Casipit CL, Gewertz NM, Blau HM. Enzymatic detection of protein translocation. Nat Methods. 2005; 2: 521-7. PMID: 15973423

16. Joffre OP, Segura E, Savina A, Amigorena S. Cross-presentation by dendritic cells. Nat Rev Immunol. 2012; 12: 557-69. PMID: 22790179

17. Azevedo MF, Nie CQ, Elsworth B, Charnaud SC, Sanders PR, Crabb BS et al. Plasmodium falciparum transfected with ultra bright NanoLuc luciferase offers high sensitivity detection for the screening of growth and cellular trafficking inhibitors. PLoS One. 2014; 9: e112571. PMID: 25392998

18. Dixon AS, Schwinn MK, Hall MP, Zimmerman K, Otto P, Lubben TH et al. NanoLuc complementation reporter optimized for accurate measurement of protein interactions in cells. ACS Chem Biol. 2016; 11: 400-8. PMID: 26569370

19. Knuepfer E, Rug M, Klonis N, Tilley L, Cowman AF. Trafficking of the major virulence factor to the surface of transfected $P$. falciparum-infected erythrocytes. Blood. 2005; 105 : 4078-87. PMID: 15692070

20. Llora-Batlle O, Tinto-Font E, Cortes A. Transcriptional variation in malaria parasites: why and how. Brief Funct Genomics. 2019; 18: 329-41. PMID: 31114839

21. Cortes A, Carret C, Kaneko O, Yim Lim BY, Ivens A, Holder AA. Epigenetic silencing of Plasmodium falciparum genes linked to erythrocyte invasion. PLoS Pathog. 2007; 3: e107. PMID:17676953

22. Iriko H, Kaneko O, Otsuki H, Tsuboi T, Su XZ, Tanabe K et al. Diversity and evolution of the rhoph 1/clag multigene family of Plasmodium falciparum. Mol Biochem Parasitol. 2008; 158(1): 11-21. PMID: 18155305

23. Gupta A, Balabaskaran-Nina P, Nguitragool W, Saggu GS, Schureck MA, Desai SA. CLAG3 self-associates in malaria parasites and quantitatively determines nutrient uptake channels at the host membrane. mBio. 2018; 9: e02293-17. PMID: 29739907

24. Gupta A, Thiruvengadam G, Desai SA. The conserved clag multigene family of malaria parasites: essential roles in host-pathogen interaction. Drug Resist Updat. 2015; 18:47-54. PMID: 25467627

25. Nguitragool W, Rayavara K, Desai SA. Proteolysis at a specific extracellular residue implicates integral membrane CLAG3 in malaria parasite nutrient channels. PLoS One 2014; 9: e93759. PMID: 24699906 
26. Gupta A, Bokhari AAB, Pillai AD, Crater AK, Gezelle J, Saggu G et al. Complex nutrient channel phenotypes despite Mendelian inheritance in a Plasmodium falciparum genetic cross. PLoS Pathog. 2020; 16: e1008363. PMID: 32069335

27. Pillai AD, Nguitragool W, Lyko B, Dolinta K, Butler MM, Nguyen ST et al. Solute restriction reveals an essential role for clag3-associated channels in malaria parasite nutrient acquisition. Mol Pharmacol. 2012; 82: 1104-14. PMID: 22949525

28. Ito D, Schureck MA, Desai SA. An essential dual-function complex mediates erythrocyte invasion and channel-mediated nutrient uptake in malaria parasites. Elife. 2017; 6:e23485. PMID: 28221136

29. Ben CR, Lentini G, Soldati-Favre D. Biogenesis and discharge of the rhoptries: Key organelles for entry and hijack of host cells by the Apicomplexa. Mol Microbiol. 2021; 115: 453-65. PMID: 33368727

30. Schureck MA, Darling JE, Merk A, Shao J, Daggupati G, Srinivasan P et al. Malaria parasites use a soluble RhopH complex for erythrocyte invasion and an integral form for nutrient uptake. Elife 2021; 10: e65282. PMID: 33393463

31. Ling IT, Florens L, Dluzewski AR, Kaneko O, Grainger M, Yim Lim BY et al. The Plasmodium falciparum clag9 gene encodes a rhoptry protein that is transferred to the host erythrocyte upon invasion. Mol Microbiol. 2004; 52: 107-18. PMID: 15049814

32. Kutner S, Baruch D, Ginsburg H, Cabantchik ZI. Alterations in membrane permeability of malaria-infected human erythrocytes are related to the growth stage of the parasite. Biochim Biophys Acta. 1982; 687: 113-7. PMID: 0007041976

33. Jackson KE, Spielmann T, Hanssen E, Adisa A, Separovic F, Dixon MW et al. Selective permeabilization of the host cell membrane of Plasmodium falciparum-infected red blood cells with streptolysin O and equinatoxin II. Biochem J. 2007; 403: 167-75. PMID: 17155936

34. Zhang Q, Ma C, Oberli A, Zinz A, Engels S, Przyborski JM. Proteomic analysis of exported chaperone/co-chaperone complexes of $P$. falciparum reveals an array of complex protein-protein interactions. Sci Rep. 2017; 7:42188. PMID: 28218284

35. Cobbold SA, Santos JM, Ochoa A, Perlman DH, Llinas M. Proteome-wide analysis reveals widespread lysine acetylation of major protein complexes in the malaria parasite. Sci Rep. 2016; 6:19722. PMID: 26813983

36. Pease BN, Huttlin EL, Jedrychowski MP, Talevich E, Harmon J, Dillman T et al. Global analysis of protein expression and phosphorylation of three stages of Plasmodium falciparum intraerythrocytic development. J Proteome Res. 2013; 12: 4028-45. PMID: 23914800 
542 37. Ribeiro JM, Garriga M, Potchen N, Crater AK, Gupta A, Ito D et al. Guide RNA selection 543 for CRISPR-Cas9 transfections in Plasmodium falciparum. Int J Parasitol. 2018; 48:825544 32. PMID: 29906414

545 38. Bokhari AA, Solomon T, Desai SA. Two distinct mechanisms of transport through the 546 plasmodial surface anion channel. J Membr Biol. 2008; 226: 27-34. PMID: 19050955 


\section{Figure Legends}

\section{Fig. 1. Design and production of host membrane-exposed split-reporter antigens. (A)}

550 Schematic shows native and modified CLAG3 topology at the host erythrocyte surface (left and

551 right top images, respectively). The native HVR sequence is replaced by varied HiBiT reporter

552

553

554

555

556

557

558

559

560

561

562

563

564

565

566

567

568

569

570

571

cassettes. Engineered lines are shown with ribbon diagrams at the bottom. (B) Immunoblots of matched total cell lysates from indicated lines, probed with antibody against a C-terminal CLAG3 epitope (top) or with LgBiT (bottom). 8-1trunc is recognized by LgBiT but not by antiCLAG3. (C) Immunoblots showing recognition of HA-tagged lines with anti-HA and LgBiT. (D) Indirect immunofluorescence assays (IFA) of indicated proteins in wild-type (WT) and transfected lines. In schizonts (top panels), CLAG3 colocalizes with RhopH3 in apical rhoptries (puncta) in $8-1 H A$, but has a more diffuse distribution in $8-1 H A$ trunc. IFA with trophozoitestage parasites (bottom panels) reveals normal export of CLAG3 and colocalization with RhopH3 at the host membrane in $8-1 H A$ but a reduced signal with failed export in 8 -1HAtrunc.

Scale bars, $5 \mathrm{~mm}$. (E) Immunoblots showing stage-specific CLAG3 abundance in indicated lines (top row, probed with $\mathrm{LgBiT}$ ). The truncated protein in 8-1HAtrunc is detected in schizonts (S) but not rings or trophozoites (R and T). Bottom row, aldolase loading control. (F) Silver-stained gel showing co-immunoprecipitation using anti-HA beads and indicated parasite lysates. WT, 8-1, and 8-1trunc represent no-HA negative controls. Yellow 1, 2, and 3 labels indicate CLAG3, RhopH2, and RhopH3, respectively. (G) Immunoblots using eluates from panel F, probed with anti-RhopH3 and anti-HA for the CLAG3 bait protein.

Fig. 2. Faithful tracking of host membrane insertion. (A) Schematic showing parasite developmental stages and host membrane insertion-dependent luminescence. After invasion, the tagged CLAG3 protein is deposited into the parasitophorous vacuole before export and eventual 
572 insertion at the host membrane. Interaction between extracellular $\mathrm{LgBiT}$ and the surface-

573 exposed HiBiT tag on 8-1 yields luminescence at mature parasite stages (purple glow). (B)

574 Bioluminescence microscopy images showing undetectable signals on immature ring-infected

575 cells (left panels), but a bright luminescence signal on 8-1 infected cells at the trophozoite stage

576 (right panels). KC5 and 8-1trunc parasites yield negligible signals. Scale bars, $10 \mu \mathrm{m}$. (C)

577 "Zoom in" of a single 8-1 infected cell from panel B, showing surface distribution of

578 luminescence signal. Scale bar, $2 \mu \mathrm{m}$. (D) Luminescence kinetics over parasite development,

579 showing CLAG3 membrane insertion in 8-1 but not 8-1trunc (red and black symbols,

580 respectively; mean \pm S.E.M. of 3 replicate wells, representative of 3 independent trials).

581 Enriched synchronized early ring-infected cells seeded at $t=0$. Increased signals at $48 \mathrm{~h}$ in both

582 parasites reflect parasite egress and release of intracellular reporter protein. (E) Mean \pm S.E.M.

583 CLAG3 exposure on indicated lines at $36 \mathrm{~h}(*, P=0.03, n=3$ trials $)$, calculated as the

584 luminescence signal normalized to total signal after cell lysis. (F) Mean \pm S.E.M. luminescence 585 signals from enriched trophozoite-infected cells without and with extracellular protease treatment 586 (black and red bars, respectively; *, $P=0.005, n=5$ ).

nutrient uptake. (A) Kinetics of osmotic lysis due to sorbitol uptake by indicated lines without 
treatment. Bar graph shows quantified fractional band intensity of the cleavage product (mean \pm S.E.M. 2 independent trials). (E) Blot probed with LgBiT showing reduction in the $150 \mathrm{kDa}$ full length CLAG3 protein upon protease treatment. Bar graph, band intensities after protease treatment, normalized to $100 \%$ without protease ( 2 independent trials).

\section{Fig. 4. Addition of $\mathrm{HA}$ and $3 \times \mathrm{HA}$ tags improve reporter signal and does not compromise} host membrane insertion. (A) Mean \pm S.E.M. luminescence signals from indicated parasite clones using matched amounts of enriched trophozoite-infected cells. Red and black bars represent intact and lysed cells, respectively $\left(*, P=10^{-4}\right.$, one-way ANOVA, $n=3$ independent trials). Signals are shown after normalization of $8-1$ readings to 1.0 in each trial. Immunoblot shows representative loading control for matching protein contents from one of the three trials. (B) Mean \pm S.E.M. luminescence signals remaining after extracellular protease treatment of intact cells from indicated lines, normalized to $100 \%$ for no protease effect ${ }^{*}, P=0.0002, n=3$ 6 for each clone). (C) Anti-CLAG3 immunoblot showing C-terminal cleavage product released by extracellular pronase E treatment. (D) Bioluminescence microscopy showing increased signals from individual cells when 1HA and 3HA tags are added to the HiBit reporter. Scale bars, $5 \mu \mathrm{m}$. (E) Mean \pm S.E.M. sorbitol permeabilities for indicated parasites, determined from osmotic lysis experiments $(n=5-21$ trials each). (F) $\%$ of transport resistant to treatment with extracellular pronase E ( $n=4-11$ trials). Channel-mediated transport is modestly affected in these lines.

\section{Fig. 5. Large reporter inserts reveal complex regulation of CLAG3 insertion at the host}

membrane. (A) To-scale schematic showing multi-HiBiT inserts and sizes of linkers between 
618

619

620

621

622

623

624

625

626

627

628

629

630

631

632

633

634

635

636

637

638

639

640

elements. The parasite names reflect the predominant linker size (in residues) followed by the number of introduced HiBiTs. (B) Anti-CLAG3 immunoblot confirming the expected increase in CLAG3 size with each construct. (C) PSAC activity in transfected clones. Top left, osmotic lysis kinetics for the KC5 parent (black), 8-1 (red), 8-2 (blue), and 8-1trunc (grey). Right, mean \pm S.E.M. apparent sorbitol permeability coefficients for indicated lines; red dotted line, mean for the $\mathrm{C} 3 h-K O$ knockout line. Note that all multi-HiBiT inserts have permeabilities indistinguishable from those of the CLAG3 knockout. Bottom left, 8-2 osmotic lysis kinetics with and without protease treatment (red and black, respectively), showing that transport is not protease sensitive in this line. Bottom right, mean \pm S.E.M. $\%$ of transport resistant to extracellular pronase $\mathrm{E}$ treatment. In contrast to the CLAG3 export competent $\mathrm{KC} 5$ and 8-1 lines, transport linked to the multi-HiBiT inserts is resistant to extracellular pronase. (D) Microscopy images showing rare luminescent cells in the $8-5$ reporter line. Jitter plot shows single cell intensities of indicated lines. Break points were selected based on the range of intensities in the $\mathrm{KC} 5$ and $8-1$ controls. Note that while most cells are negative in the multiHiBiT constructs, a small number have very high signals in each reporter line. (E) 2D plot showing molecular weight (MW) and net charge at $\mathrm{pH} 7.4$ for the CLAG3 HVR region from 38 available sequences (black circles) and the indicated engineered substitutions (red symbols).

\section{Supporting Figure Legends}

S1 Fig. Construction and validation of reporter protein for host membrane insertion. (A) Sequences of the modified CLAG3 locus in 8-1 and 8-1HA reporter lines aligned with the parental KC5 CLAG3h and CLAG3.1 and CLAG3.2 from divergent $P$. falciparum lines (7G8 and Dd2 from Brazil and Indochina, respectively). The HVR sequences from wild-type lines is 
641 highlighted in grey; introduced HiBiT is shown with magenta highlight. HA tag is underlined;

642 the sites where stop codons were introduced to produce 8-1trunc and 8-1HAtrunc are marked

643 with red arrows. Identical and conserved residues that flank the HVR are in red and blue,

644 respectively. Note the length polymorphism in native HVR sequences; the modification to

645 produce 8-1HA increases the length of this extracellular motif. (B) IFA of trophozoite-stage

646 parasites probed with a CLAG3-specific antibody directed against a C-terminal epitope [9].

647 Scale bar, $5 \mu \mathrm{m}$. Notice unchanged export and colocalization with RhopH3 in $8-1$ and $8-1 H A$

648 parasites. (C) Modified CLAG3 locus sequence in the 8-1-3HA parasite with color-coding as in

649 panel $\mathbf{A}$. The $3 \times \mathrm{xHA}$ tag is underlined.

650

S2 Fig. Trafficking and membrane insertion for large multi-HiBiT insertions into the

652 CLAG3 HVR. IFA micrographs of indicated lines at the schizont and trophozoite stages (panels

653 A and B, respectively), showing that each construct is faithfully trafficked to rhoptry organelles

654 in schizonts, but that the multi-HiBiT lines do not export CLAG3 to the host membrane of most

655 trophozoite-infected cells. Scale bars, $5 \mu \mathrm{m}$. (C) Anti-CLAG3 immunoblot showing that

656 chymotrypsin releases a $\sim 35 \mathrm{kDa}$ cleavage product in $\mathrm{KC} 5$ and $8-1$ parasites, but that cleavage

657 is not detected in studies with the indicated multi-HiBiT lines. 
A

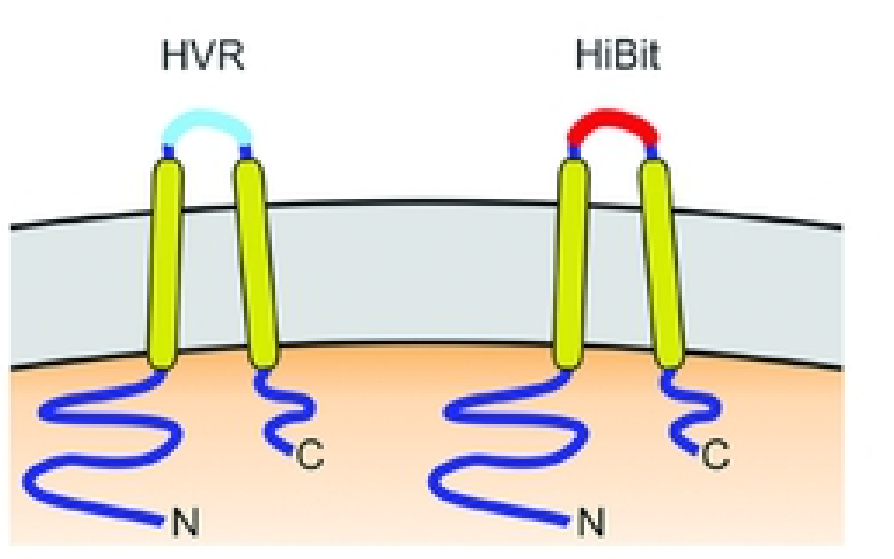

B $\quad \frac{\alpha \text {-CLAG3 }}{\text { KC5 8-1 8-1trunc }}$

$\square$ HVR $\square$ linker

$\square$ TM $\square \mathrm{HA}$

$\square$ HiBit $\square$ Stop

KC5 CLAG3h

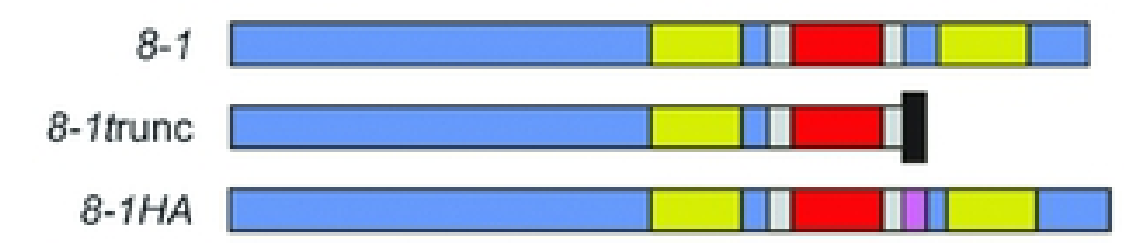

8-1HAtrunc

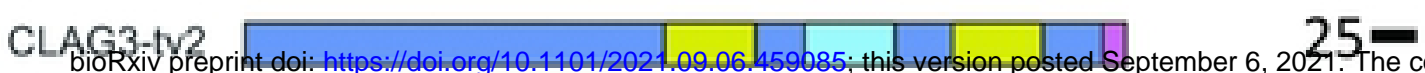

(which was not certified by peer review) is the author/funder. This article is a US Government work. It is not subject to copyright under 17 USC

$150 \mathrm{KDa}=$

LgBiT

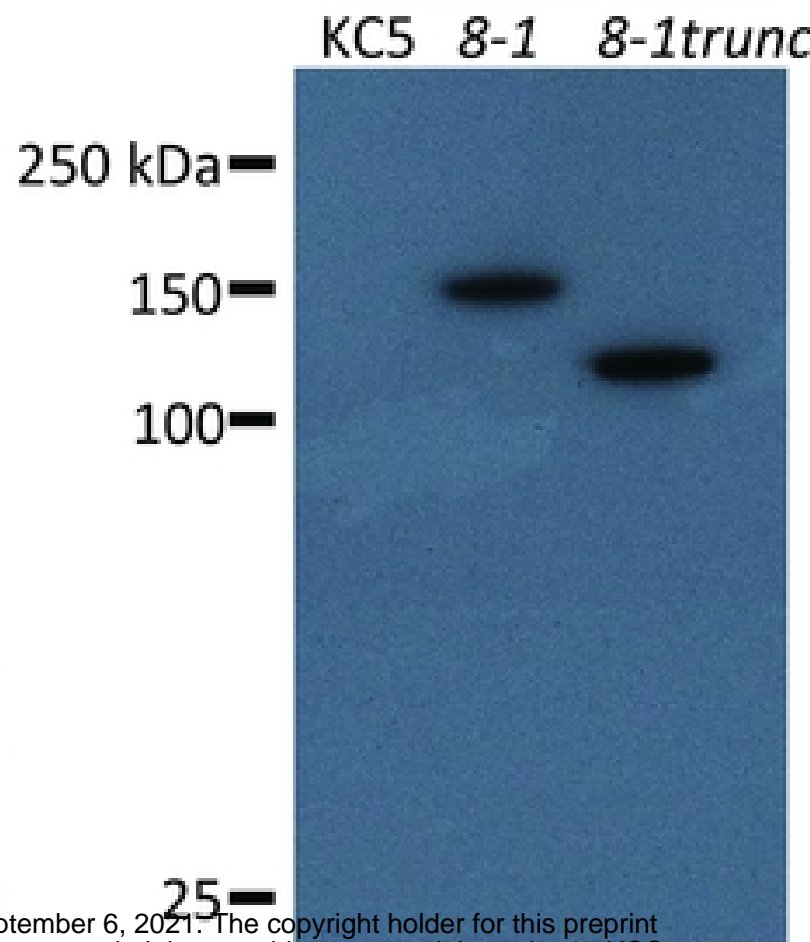

C

$\alpha-\mathrm{HA}$

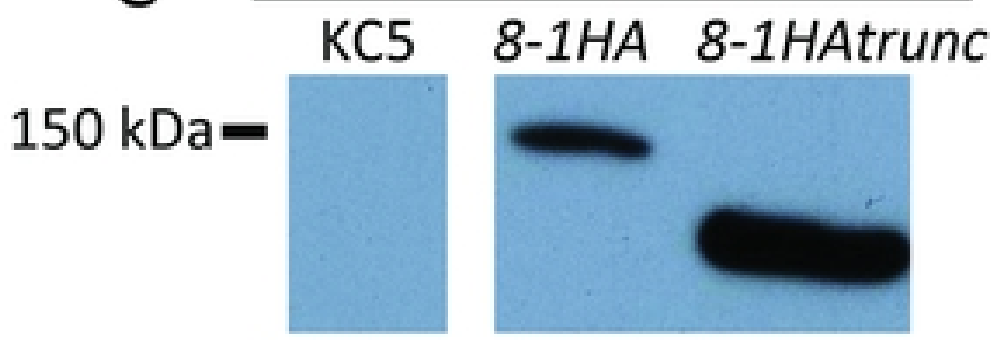

$150 \mathrm{kDa}-$

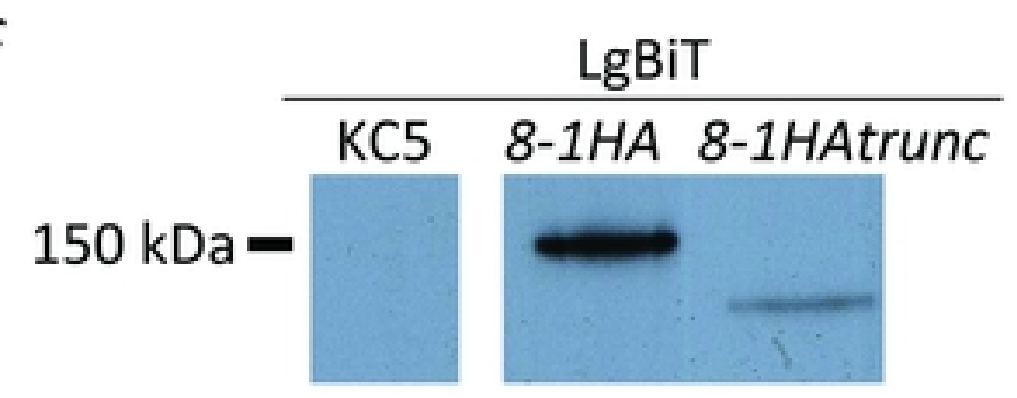

D

8-1HAtrunc

DIC

KC5

8-1HA
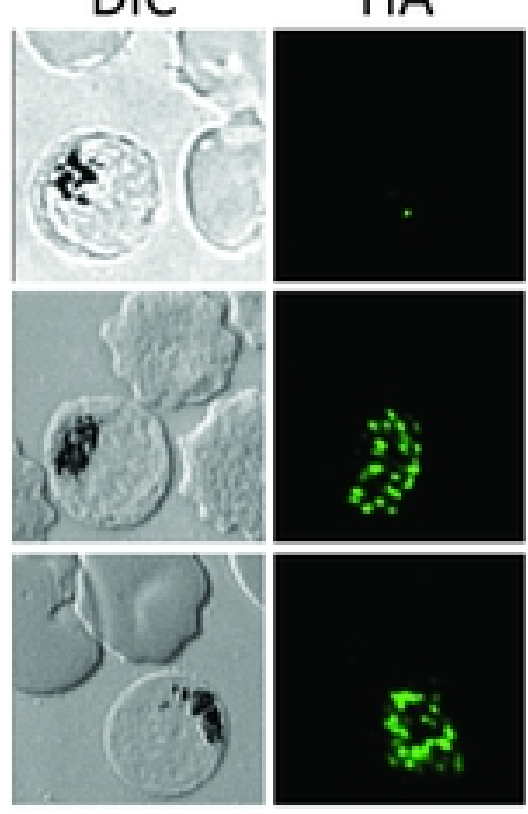

DIC

KC5

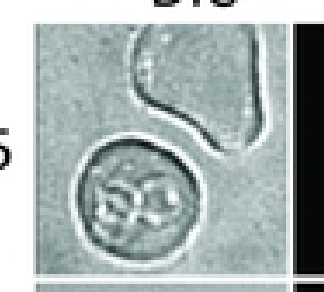

8-1HA

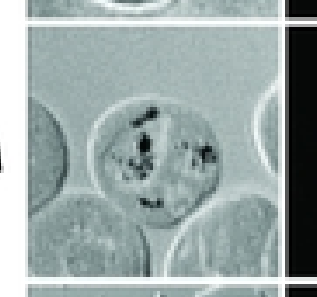

\begin{abstract}
HA
\end{abstract}

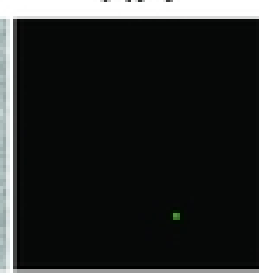

RhopH3

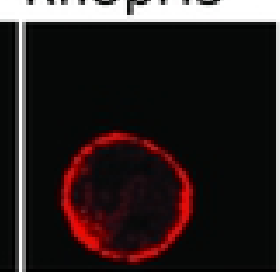

Merge
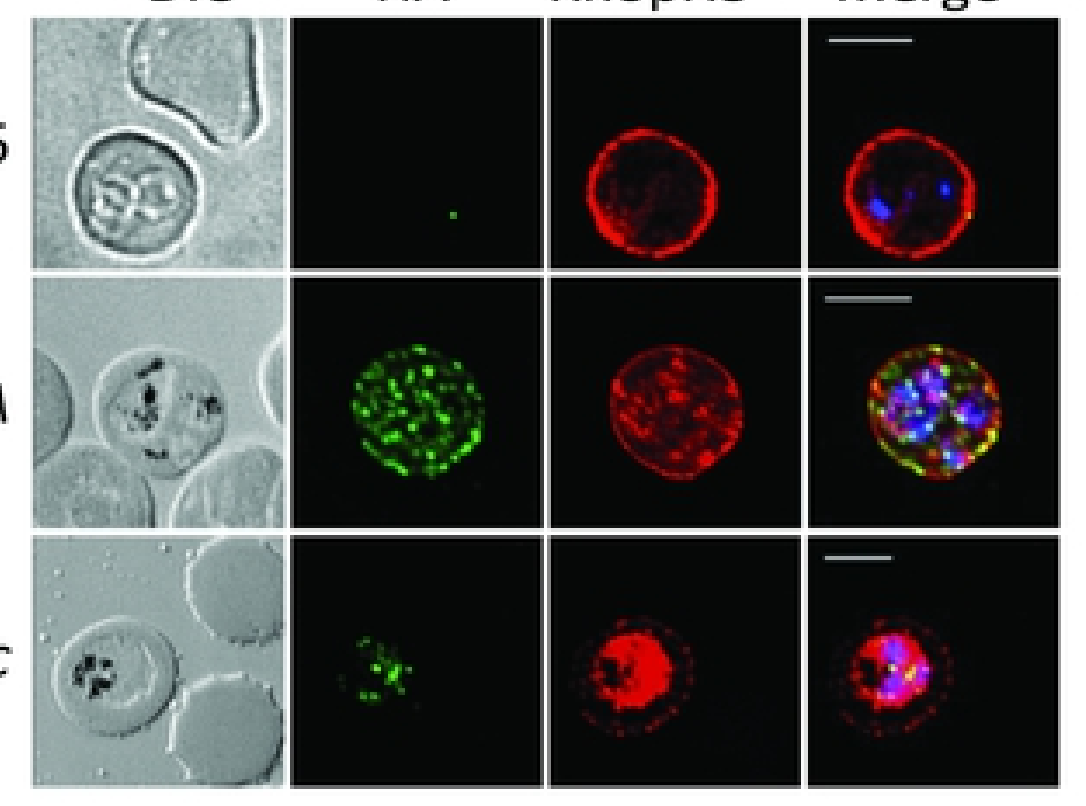

E

8-1HAtrunc

E

Figure 1

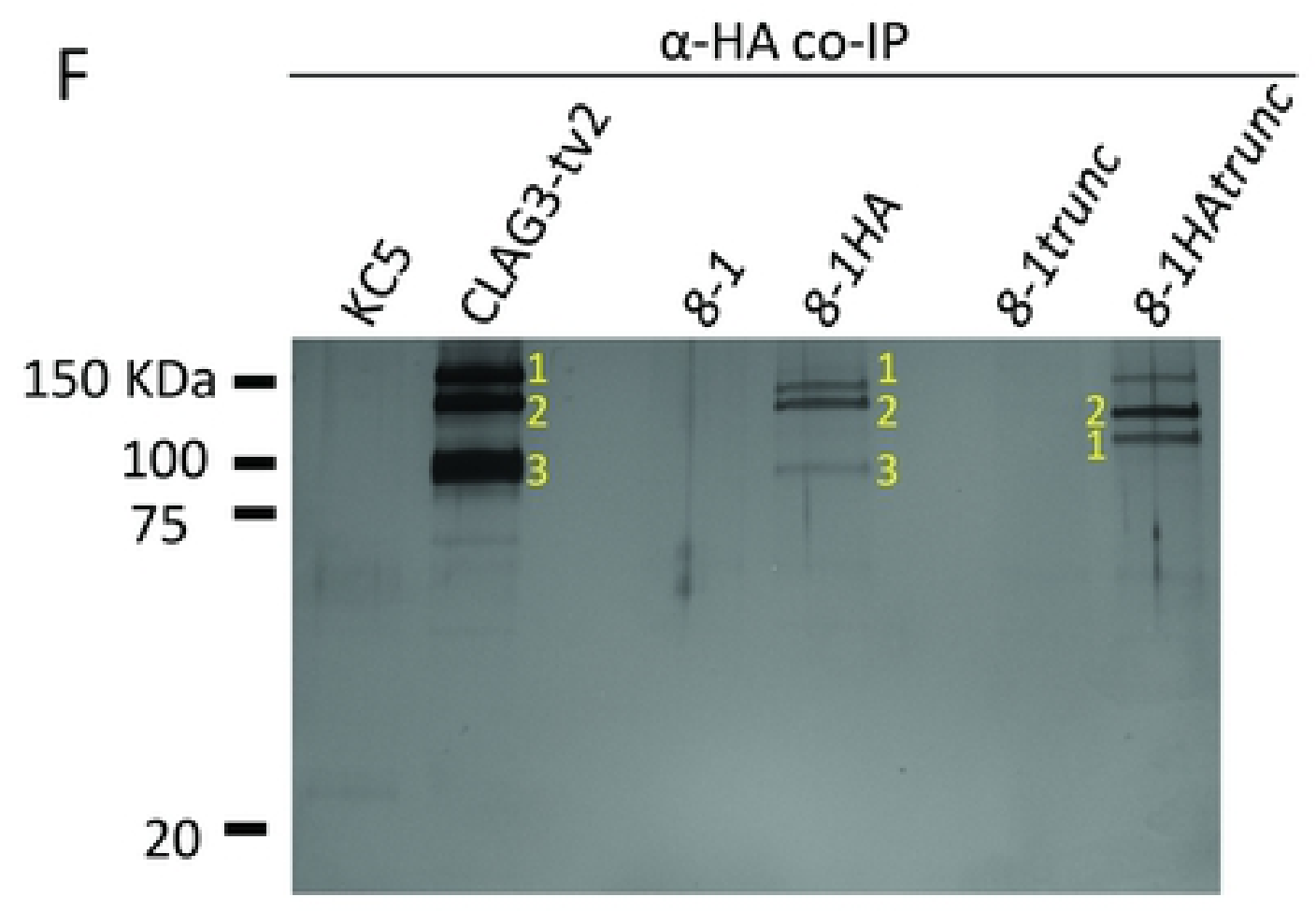

G

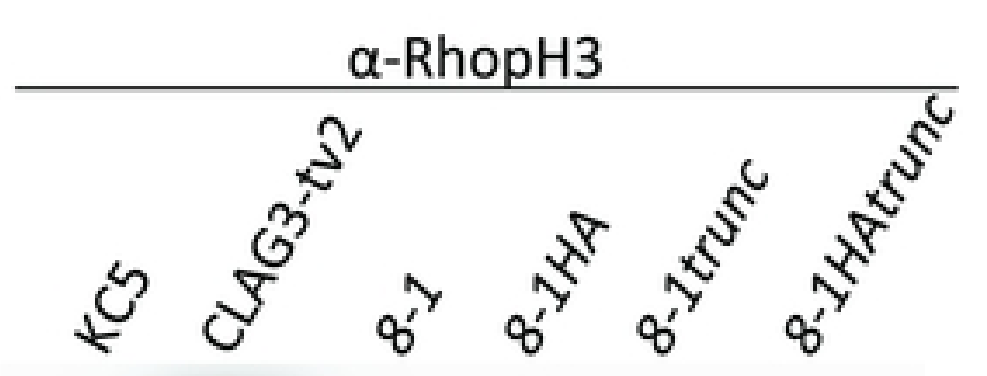

$100 \mathrm{kDa}-$

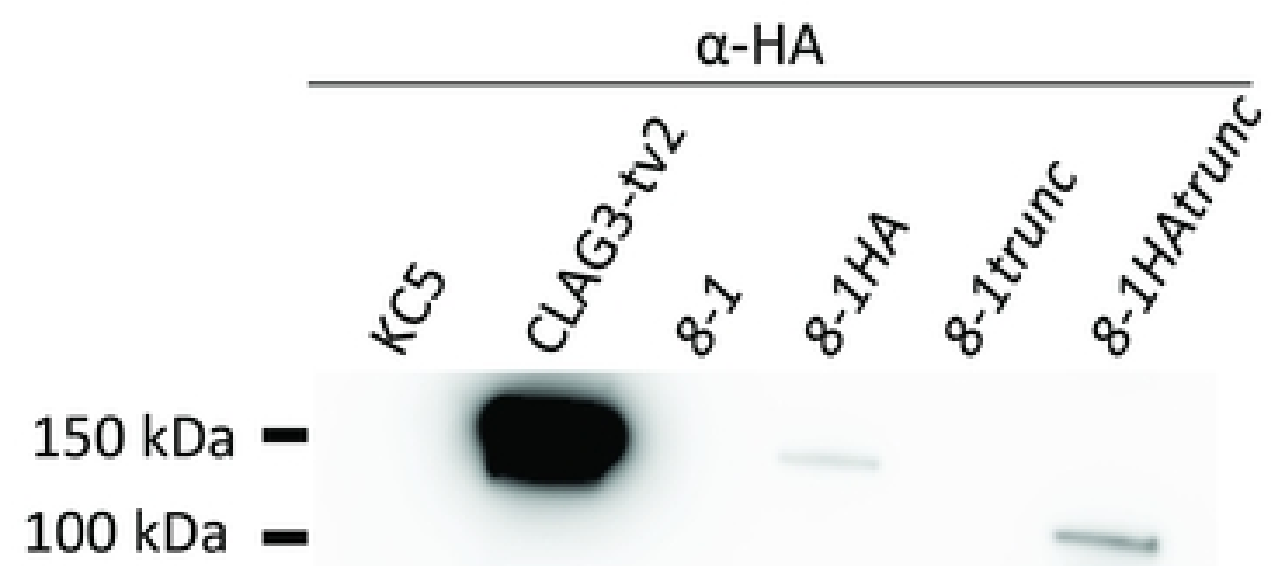


A
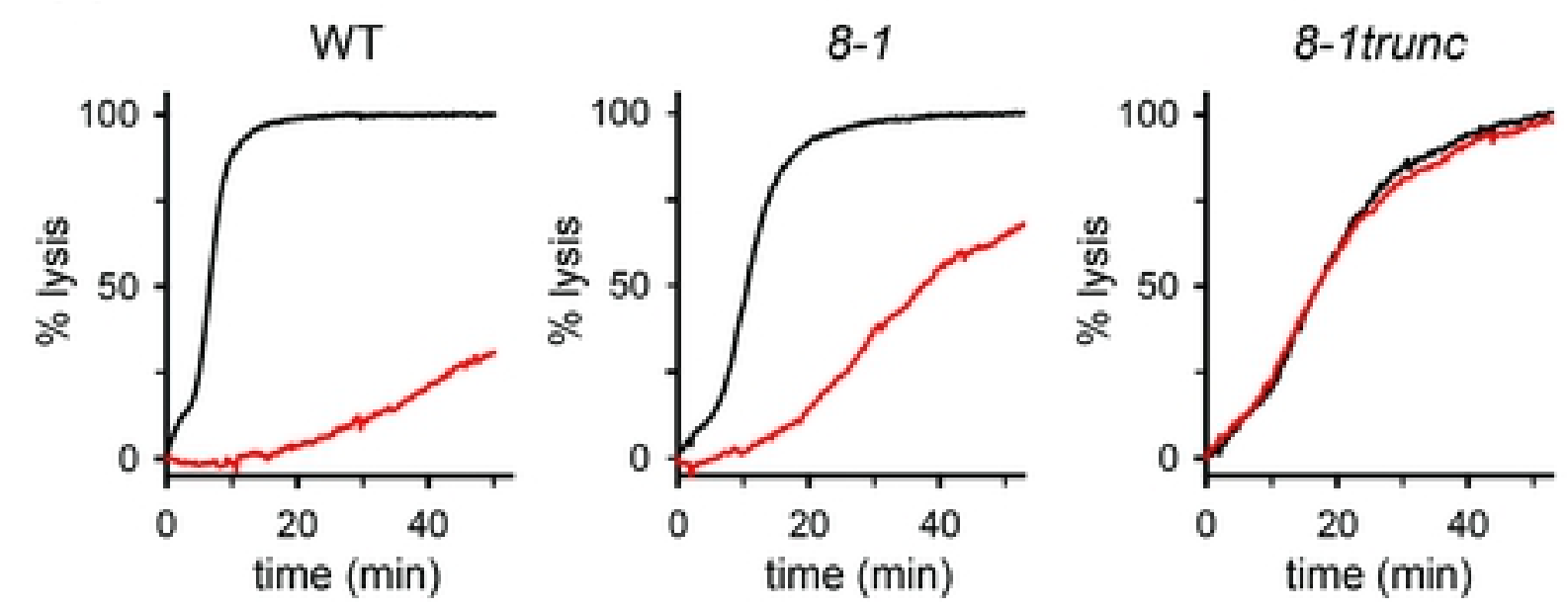

B

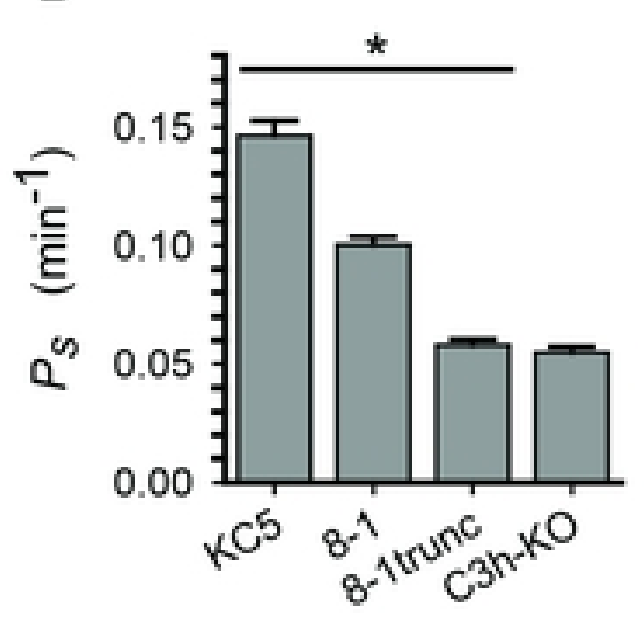

C

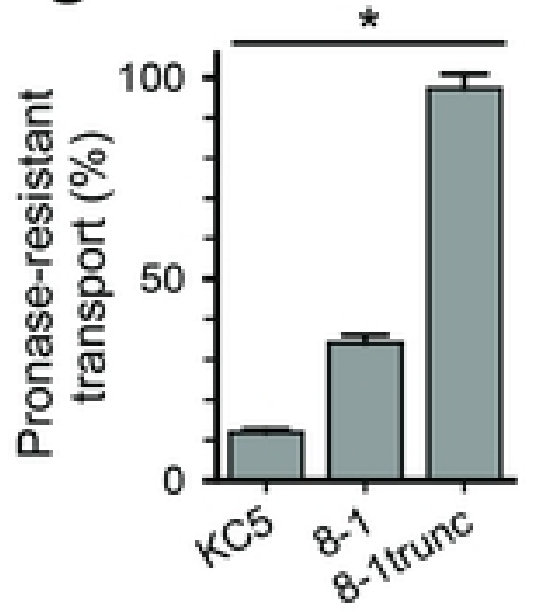

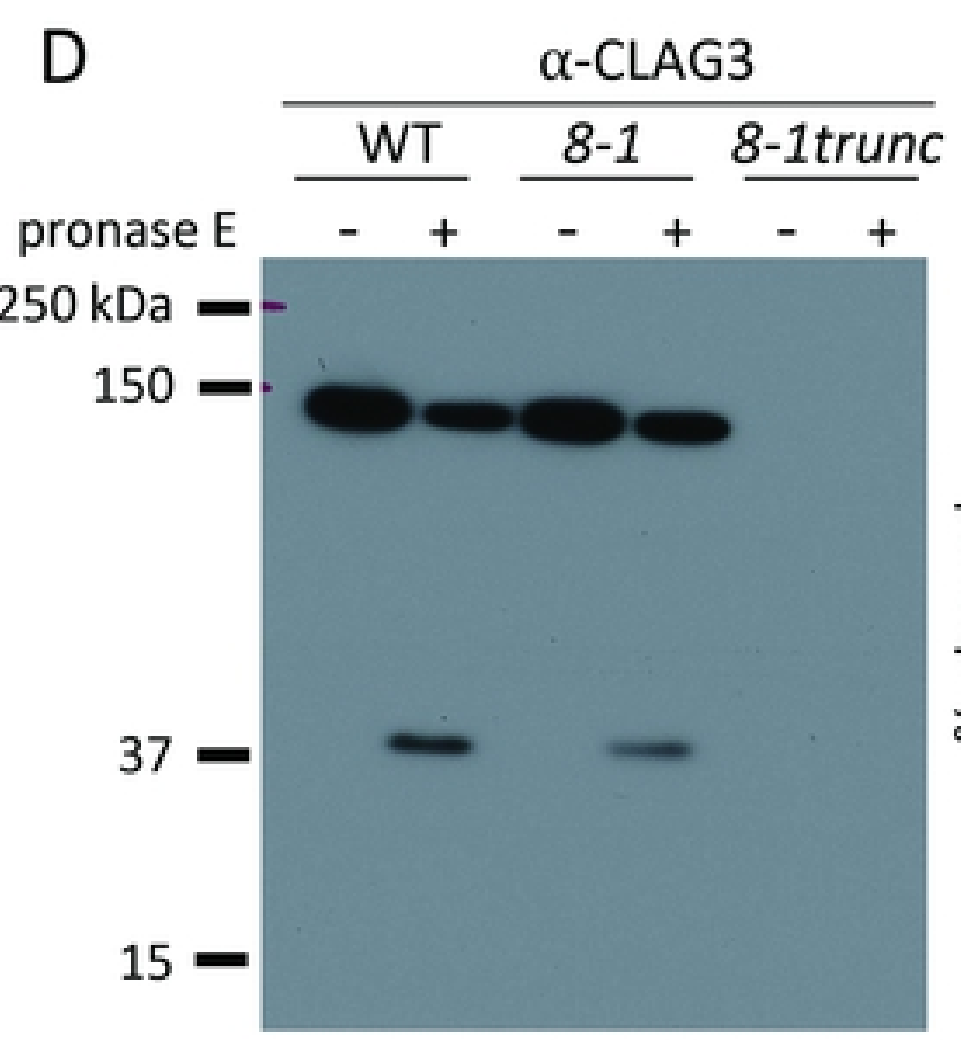
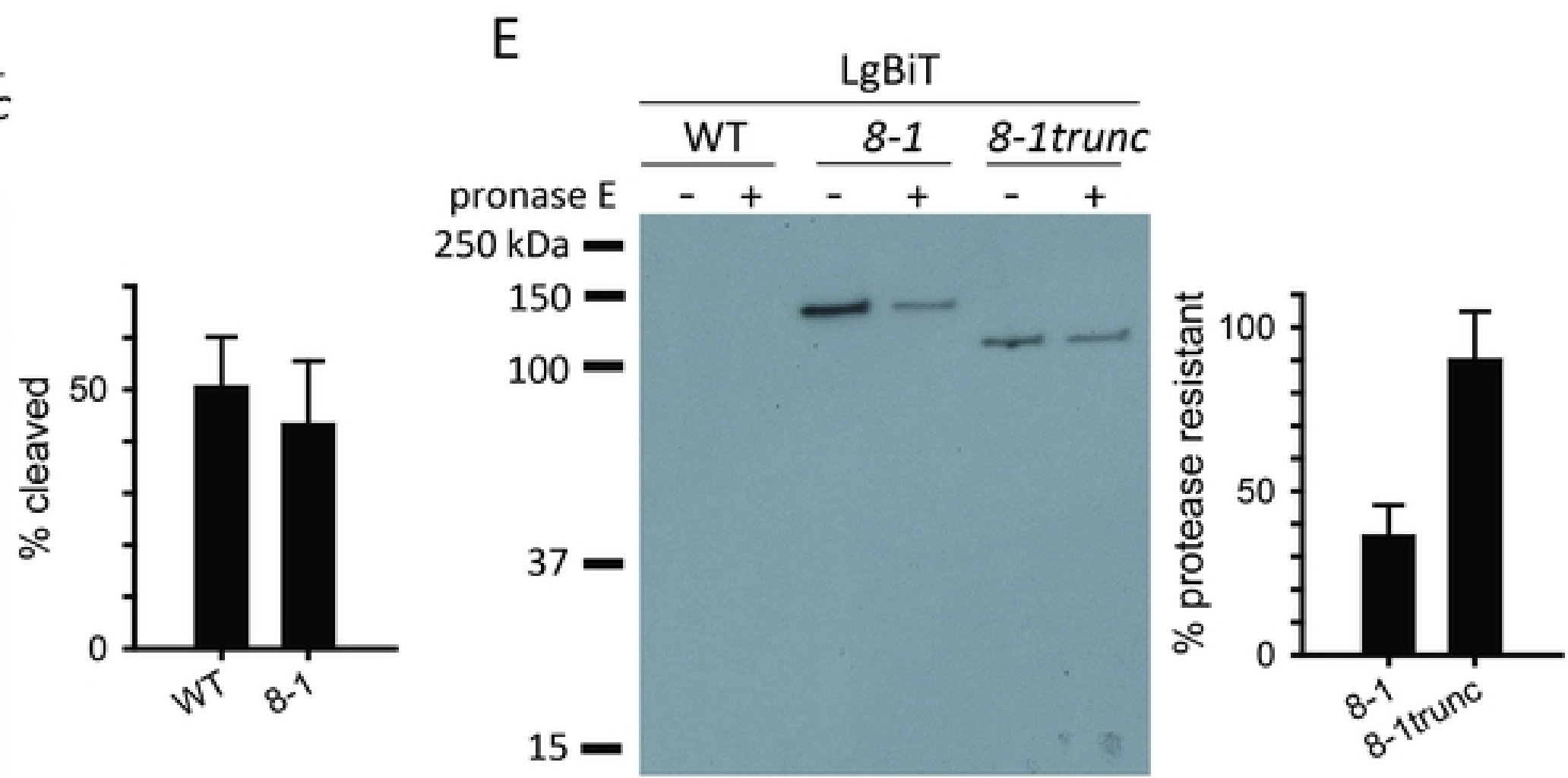

Figure 3 
A

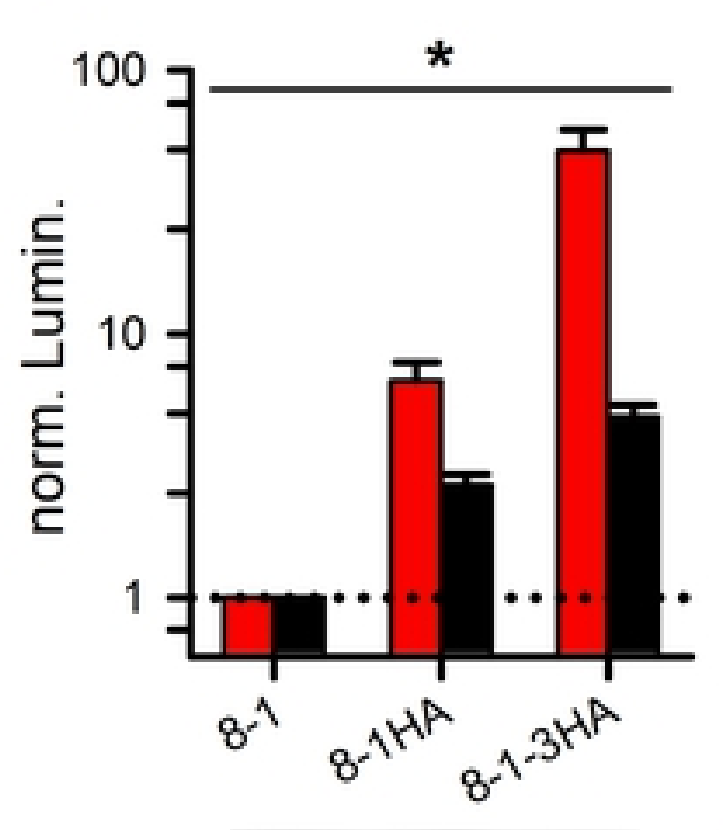

B

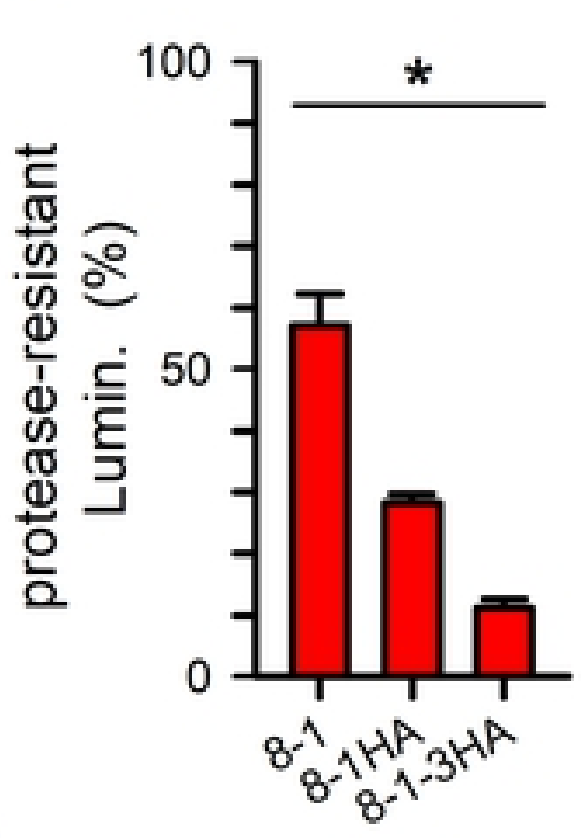

E

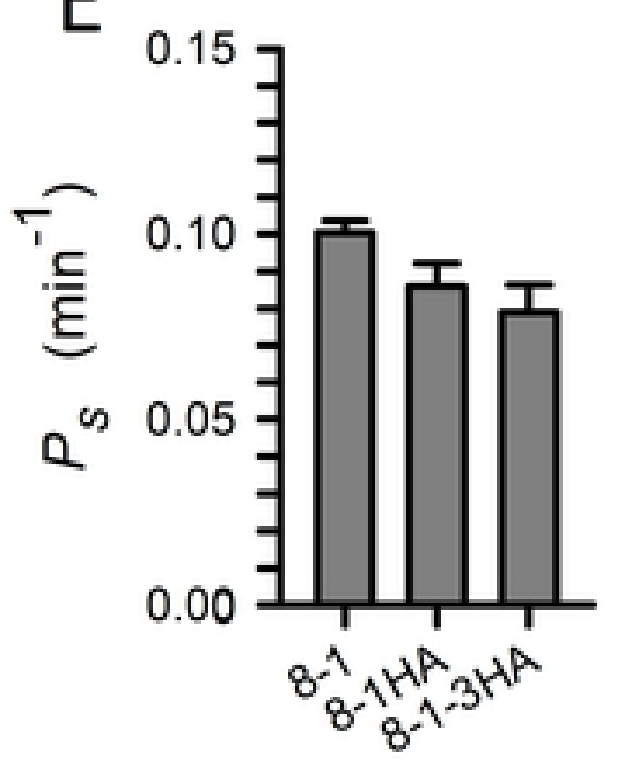

C

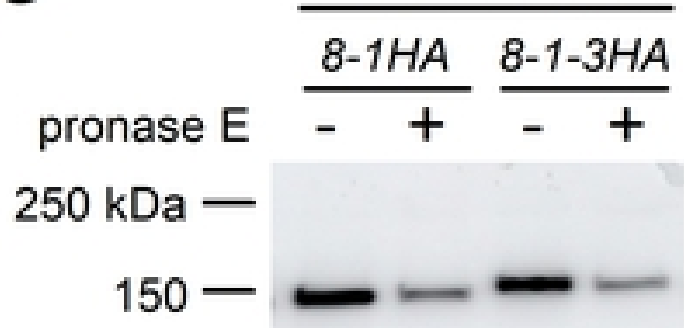

$37-$

$15-$

$\mathrm{F}$

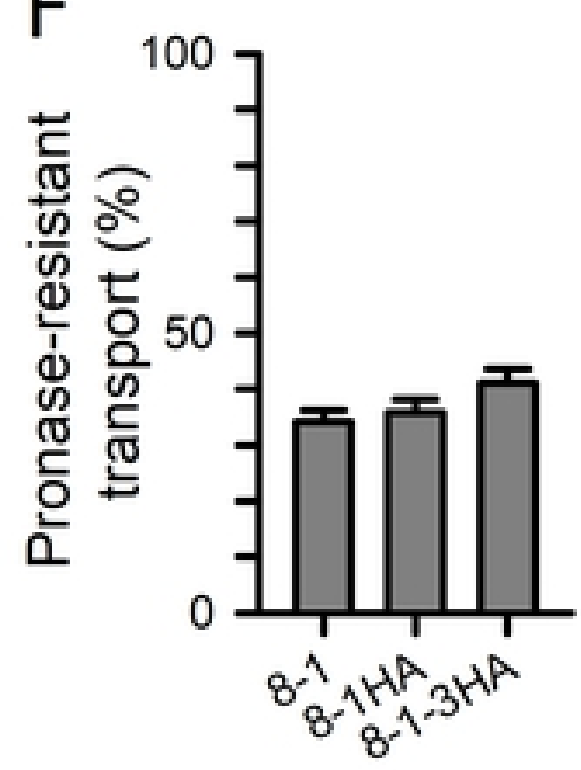

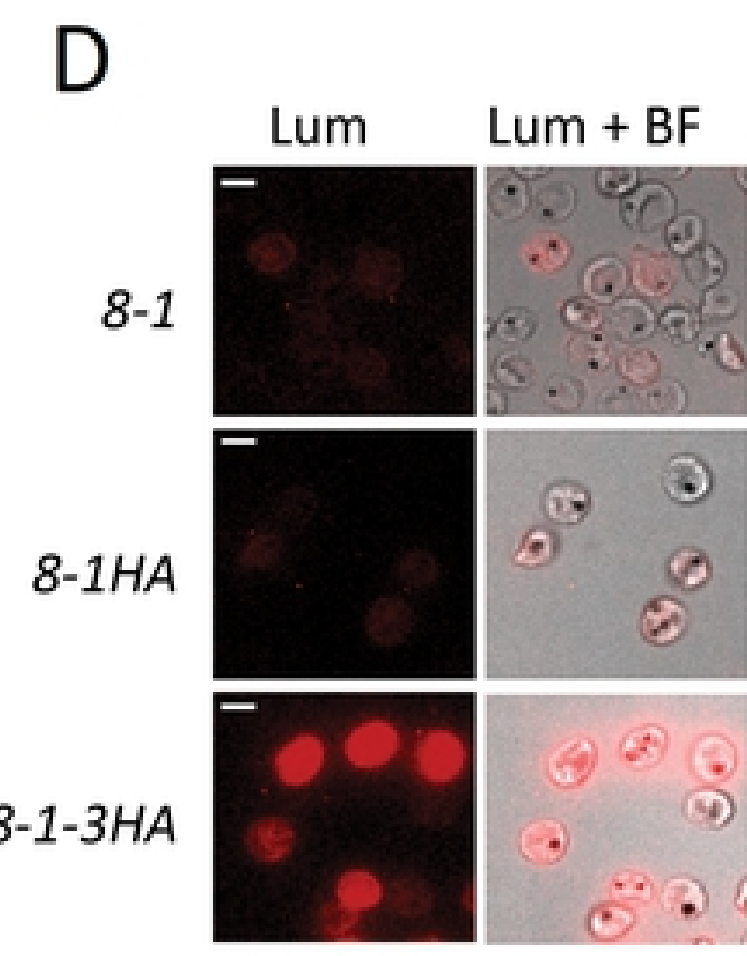

$\alpha$-CLAG3

$\alpha$-aldolase

Figure 4 


\section{A}

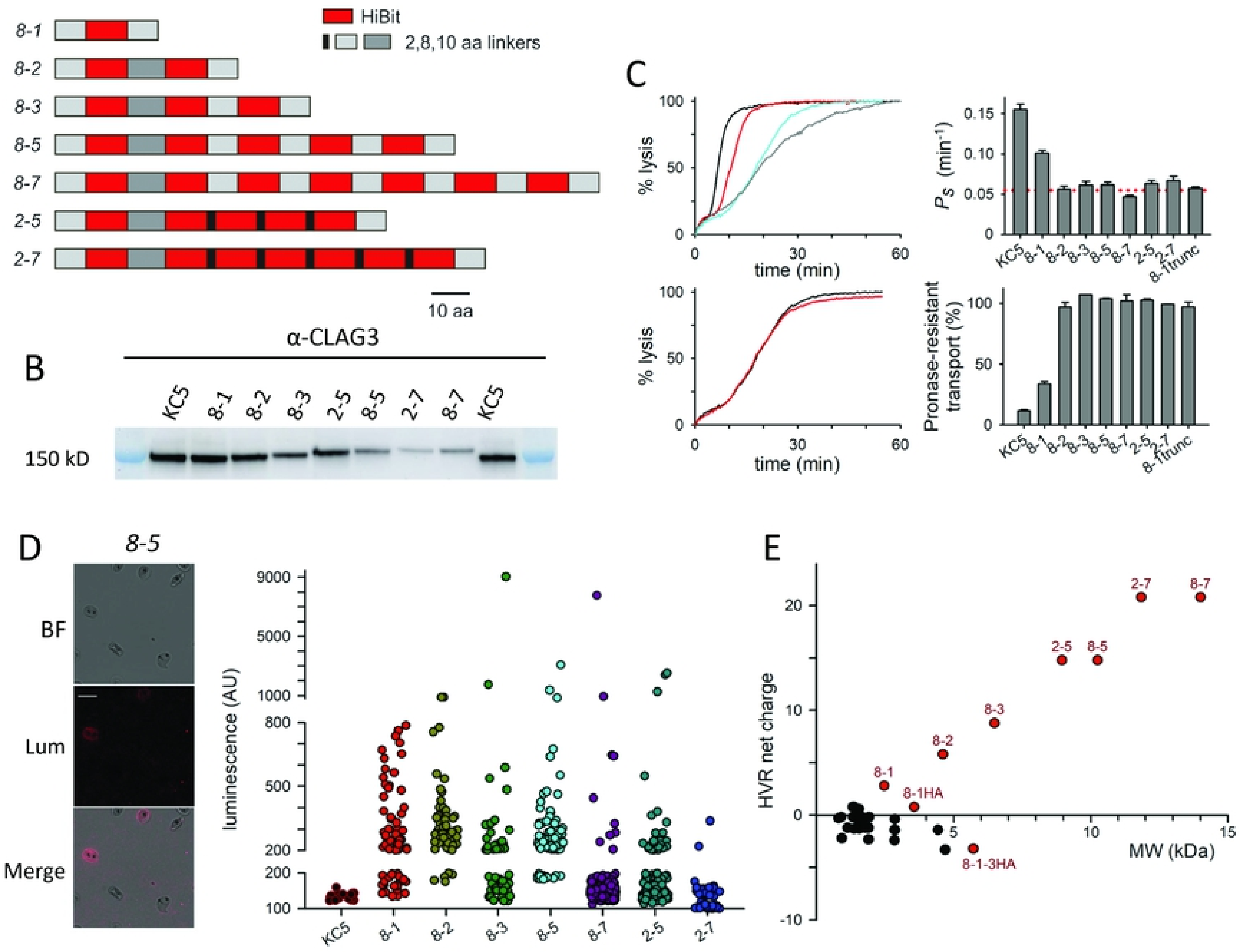

Figure 5 